\title{
Comparative evaluation of ALBI, MELD, and Child-Pugh scores in prognosis of cirrhosis: is ALBI the new alternative?
}

\author{
Maria Fragaki ${ }^{a}$, Dimitra Sifaki-Pistolla ${ }^{b}$, Eleni Orfanoudaki ${ }^{a}$, Elias Kouroumalis ${ }^{a}$ \\ University Hospital of Heraklion and University of Crete, Medical School; University of Crete, Medical School, Crete, \\ Greece
}

Abstract

Background The existence of reliable prognostic indices is of paramount importance in the management of cirrhosis. Both the model for end-stage liver disease (MELD) score and the older Child-Pugh (CP) scores are widely used. The albumin-bilirubin (ALBI) score, initially used in hepatocellular carcinoma, has not been thoroughly investigated in cirrhosis. The aim of this study was to compare the prognostic accuracy of ALBI, MELD, MELD with sodium (MELD-Na), CP, and the corrected for creatinine CP scores in a genetically homogeneous Cretan cirrhotic population.

Methods One hundred ninety-five outpatients or hospitalized cirrhotics (127 male, median age 66 years) were studied over a period of 2 years and ALBI, platelet-albumin-bilirubin, MELD, MELD-Na, CP score, and 2 types of modified CP score (CP-I and CP-II) with serum creatinine were calculated and correlated with survival.

Results ALBI had an optimum balance between sensitivity and specificity (area under the curve $0.704,95 \%$ confidence interval [CI] 0.630-0.778) compared to the other scores. In the multivariate analysis, the only factors independently associated with death were the ALBI score (hazard ratio [HR] 2.51, 95\%CI 1.69-3.73; $\mathrm{P}<0.001$ ), the MELD-Na score (HR 1.04, 95\%CI 1.00-1.09; P=0.045), and age (HR 1.05, 95\%CI 1.03-1.07; $\mathrm{P}<0.001)$. When only decompensated cirrhosis was evaluated, the multivariate analysis showed that the ALBI score (HR 3.03; 95\%CI 1.92-4.78; $\mathrm{P}<0.001$ ), and age (HR 1.05, 95\%CI 1.03-1.07; $\mathrm{P}<0.001$ ) were independently associated with death.

Conclusion ALBI score might be a better prognostic indicator of mortality in cirrhosis and given its simplicity could substitute for the CP, MELD, and MELD-Na scores.

Keywords Cirrhosis, model for end-stage liver disease, Child-Pugh, albumin-bilirubin score

Ann Gastroenterol 2019; 32 (6): 1-7

\section{Introduction}

The existence of reliable prognostic indices is of paramount importance in the management of cirrhosis. The Child-Pugh (CP) score has been used for more than 40 years. It was initially

${ }^{a}$ Department of Gastroenterology and Hepatology, University Hospital of Heraklion and University of Crete, Medical School (Maria Fragaki, Eleni Orfanoudaki, Elias Kouroumalis); ${ }^{\mathrm{b}} \mathrm{Clinic}$ of Social and Family Medicine, University of Crete, Medical School (Dimitra Sifaki-Pistolla), Crete, Greece

\section{Conflict of Interest: None}

Correspondence to: Maria Fragaki, MD, Department of Gastroenterology \& Hepatology, University Hospital of Heraklion, Building A- $4^{\text {th }}$ Floor, Voutes, Heraklion 71110, Crete, Greece, e-mail:mgfragaki@yahoo.gr

Received 9 April 2019; accepted 31 July 2019; published online 26 September 2019

DOI: https://doi.org/10.20524/aog.2019.0417 proposed to assess the outcomes of cirrhotic patients after surgery for portal hypertension and gradually gained wider acceptance [1-3]. Two modified types of CP score encompassing serum creatinine have also been evaluated [4]. The model for end-stage liver disease (MELD) score was first applied to patients undergoing transjugular intrahepatic portosystemic shunts [5], but since 2002 it has been used for assessing candidates for liver transplantation [6]. MELD-Na, a modified MELD score incorporating serum sodium, has been used for survival prediction, taking account of the significance of hyponatremia in early mortality from cirrhosis [7-9].

The albumin-bilirubin (ALBI) score is a recently proposed and very simple score that evaluates only 2 objective parameters that are readily available for every cirrhotic patient. The ALBI score has been reported to assess liver dysfunction and prognosis in patients with hepatocellular carcinoma $[10,11]$, in patients with primary biliary cholangitis [12], in cirrhotic patients with upper gastrointestinal bleeding [13], and in various hepatitis-B virusrelated liver diseases $[14,15]$. However, its usefulness in assessing a cirrhotic cohort of patients has not been adequately evaluated. 
The aim of this study was to compare the prognostic accuracy of ALBI, platelet-albumin-bilirubin (PALBI), MELD, MELD-Na, CP, and CP corrected for creatinine scores in a genetically homogeneous Cretan cirrhotic population.

\section{Patients and methods}

Data on 195 consecutive cirrhotic patients either attending the liver outpatient clinic or hospitalized in the gastroenterology wards within a 2-year period were retrospectively analyzed. The study was approved by the Institutional Ethics Review Board of the Hospital and written consent was provided by all patients. Cirrhosis was determined either by liver biopsy (52\%) or by compatible imaging and clinical and endoscopic findings (esophageal varices, ascites). Cirrhosis was classified as decompensated when ascites, variceal hemorrhage or hepatic encephalopathy were recorded.

Age, sex, cause of cirrhosis, compensation status, age at diagnosis of cirrhosis, first complications of decompensated cirrhosis, existence or appearance of hepatocellular cancer, as well as biochemical variables (creatinine, total bilirubin,

Table 1 Baseline clinical and laboratory characteristics in 195 cirrhotic patients

\begin{tabular}{|c|c|}
\hline Characteristics & Value \\
\hline Age (years), median (IQR, range) & $66(18,31-95)$ \\
\hline Male sex, n (\%) & $127(65.1)$ \\
\hline $\begin{array}{l}\text { Etiology of cirrhosis, n (\%) } \\
\text { Alcohol } \\
\text { Viral } \\
\text { Alcohol \& viral } \\
\text { NAFLD } \\
\text { PBC } \\
\text { Cryptogenic } \\
\text { Decompensated cirrhosis, n (\%) }\end{array}$ & $\begin{array}{c}71(36.4) \\
56(28.7) \\
16(8.2) \\
21(10.8) \\
18(9.2) \\
4(2.1) \\
142(72.8)\end{array}$ \\
\hline $\begin{array}{l}\text { Laboratory results }(\text { mean } \pm \mathrm{SD}) \\
\text { Creatinine }(\mathrm{mg} / \mathrm{dL}) \\
\text { Serum albumin }(\mathrm{g} / \mathrm{dL}) \\
\text { Total serum bilirubin }(\mathrm{mg} / \mathrm{dL}) \\
\text { International normalized ratio }\end{array}$ & $\begin{array}{c}1.09(0.27) \\
3.3(0.72) \\
2.8(3.49) \\
1.39(0.38)\end{array}$ \\
\hline Ascites, n (\%) & $99(50.8)$ \\
\hline Hepatic encephalopathy, n (\%) & $33(16.9)$ \\
\hline MELD score, median (IQR, range) & $12(9,6-30)$ \\
\hline MELD-Na score, median (IQR, range) & $15(11,3-33)$ \\
\hline CP score, median (IQR, range) & $7(4,5-13)$ \\
\hline CP score I, median (IQR, range) & $8(5,5-17)$ \\
\hline CP score II, median (IQR, range) & $7(5,5-15)$ \\
\hline ALBI, median (IQR, range) & $-2.68(1.23,-4.25$ to -0.64$)$ \\
\hline PALBI, median (IQR, range) & $-2.55(0.91,-3,17$ to -0.21$)$ \\
\hline
\end{tabular}

NAFLD, non-alcoholic fatty liver disease; $P B C$, primary biliary cholangitis; $I Q R$, interquartile range; $M E L D$, model for end-stage liver disease;

MELD-Na, model for end-stage liver disease with sodium; CP Child-Pugh; ALBI, albumin-bilirubin score; PALBI, platelet-albumin-bilirubin score albumin, international normalized ratio, prothrombin time, sodium, potassium, alkaline phosphatase, $\gamma$-glutamyl transferase, transaminases) were recorded for all patients.

Patients were followed-up for a median of 27.2 months (interquartile range [IQR] 46.9, 95\% confidence interval [CI] 0-105.6). Three patients were lost to follow up. Every cirrhotic patient was followed-up in our screening program for hepatocellular carcinoma, which involves undergoing an ultrasound examination every 6 months with a-fetoprotein levels and contrast-enhanced ultrasound with SonoVue every year. The CP, MELD, MELD-Na, ALBI, and PALBI scores were assessed for every patient at first examination $[1,2,11,16]$. The PALBI score was calculated as:

PALBI $=\left(2.02 \times \log _{10}\right.$ bilirubin $)+\left(-0.37 \times\left(\log _{10} \text { bilirubin }\right)^{2}\right)$ $+(-0.04 \times$ albumin $)+\left(-3.48 \times \log _{10}\right.$ platelets $)+(1.01 \times$ $\left.\left(\log _{10} \text { platelets }\right)^{2}\right)$ [17].

In addition, 2 types of modified CP score (CP-I and CP-II) with serum creatinine as the sixth variable were also calculated:

Table 2 Comparisons of the areas under the ROC curve for CP, CP-I, CP-II, MELD, MELD-Na, ALBI and PALBI scores in relation to 1-, 6-, 12-, and 24-month survival

\begin{tabular}{|c|c|c|c|c|}
\hline $\begin{array}{l}\text { Survival } \\
\text { (months) }\end{array}$ & Score & $\begin{array}{l}\text { Area under } \\
\text { ROC curve }\end{array}$ & $95 \% \mathrm{CI}$ & $\mathrm{P}$-value \\
\hline \multirow[t]{7}{*}{1} & CP score & 0.889 & $0.82-0.95$ & $<0.001$ \\
\hline & CP-I & 0.874 & $0.80-0.94$ & $<0.001$ \\
\hline & CP-II & 0.889 & $0.82-0.95$ & $<0.001$ \\
\hline & MELD & 0.874 & $0.79-0.95$ & $<0.001$ \\
\hline & $\begin{array}{l}\text { MELD-Na } \\
\text { score }\end{array}$ & 0.874 & $0.80-0.94$ & $<0.001$ \\
\hline & ALBI & 0.912 & $0.84-0.98$ & $<0.001$ \\
\hline & PALBI & 0.823 & $0.71-0.92$ & $<0.001$ \\
\hline \multirow[t]{7}{*}{6} & CP score & 0.786 & $0.71-0.85$ & $<0.001$ \\
\hline & CP-I & 0.796 & $0.72-0.86$ & $<0.001$ \\
\hline & CP-II & 0.793 & $0.72-0.86$ & $<0.001$ \\
\hline & MELD & 0.767 & $0.69-0.84$ & $<0.001$ \\
\hline & $\begin{array}{l}\text { MELD-Na } \\
\text { score }\end{array}$ & 0.795 & $0.724-0.86$ & $<0.001$ \\
\hline & ALBI & 0.785 & $0.70-0.86$ & $<0.001$ \\
\hline & PALBI & 0.737 & $0.65-0.82$ & $<0.001$ \\
\hline \multirow[t]{7}{*}{12} & CP score & 0.763 & $0.69-0.83$ & $<0.001$ \\
\hline & CP-I & 0.770 & $0.69-0.84$ & $<0.001$ \\
\hline & CP-II & 0.769 & $0.69-0.84$ & $<0.001$ \\
\hline & MELD & 0.748 & $0.67-0.81$ & $<0.001$ \\
\hline & $\begin{array}{l}\text { MELD-Na } \\
\text { score }\end{array}$ & 0.780 & $0.71-0.84$ & $<0.001$ \\
\hline & ALBI & 0.781 & $0.71-0.85$ & $<0.001$ \\
\hline & PALBI & 0.717 & $0.64-0.79$ & $<0.001$ \\
\hline \multirow[t]{7}{*}{24} & CP score & 0.751 & $0.68-0.82$ & $<0.001$ \\
\hline & CP-I & 0.753 & $0.68-0.82$ & $<0.001$ \\
\hline & CP-II & 0.754 & $0.68-0.82$ & $<0.001$ \\
\hline & MELD & 0.732 & $0.66-0.80$ & $<0.001$ \\
\hline & $\begin{array}{l}\text { MELD-Na } \\
\text { score }\end{array}$ & 0.755 & $0.68-0.82$ & $<0.001$ \\
\hline & ALBI & 0.780 & $0.71-0.84$ & $<0.001$ \\
\hline & PALBI & 0.694 & $0.62-0.77$ & $<0.001$ \\
\hline
\end{tabular}

ROC, receiver operating characteristic; CI, confidence interval; $C P$, Child-Pugh; MELD, model for end-stage liver disease; MELD-Na, model for end-stage liver disease with sodium; ALBI, albumin-bilirubin score; PALBI, platelet-albumin-bilirubin score 
CP-I (range: 5-19) was derived from the original CP score by adding 0 points for creatinine $<1.3 \mathrm{mg} / \mathrm{dL}$ and 4 points for creatinine $\geq 1.3 \mathrm{mg} / \mathrm{dL}$, according to Angermayr et al [4], while CP-II (range: 5-19) was derived from the original CP score by adding 0 points for creatinine $<1.3 \mathrm{mg} / \mathrm{dL}, 2$ points for creatinine $1.3-1.8 \mathrm{mg} / \mathrm{dL}$, and 4 points for creatinine $>1.8 \mathrm{mg} / \mathrm{dL}$. According to the proposed cutoff points for ALBI and PALBI scores, we stratified our patients into 3 groups. For ALBI score: grade 1 (score -2.60), grade 2 (>-2.60 to -1.39), and grade $3(>-1.39)$ [11]. For PALBI score: grade 1 (score $\leq-2.53)$, grade $2(>-2.52$ to -2.09$)$, and grade $3(>-2.09)[17,18]$.

\section{Statistical analysis}

Analysis was performed using the IBM SPSS 14.0 software, with statistical significance defined at $a=0.05$. All variables were checked for normality of distributions by the KolmogorovSmirnov test and the binomial test. Data were reported as mean \pm standard deviation and median, IQR. Continuous variables were compared using Student's $t$-test, the MannWhitney $U$-test or one-way analysis of variance, as appropriate. Furthermore, receiver operating characteristic (ROC) analysis was performed and the area under the curve (AUC) was calculated to assess the 6 scoring systems. Survival analysis was conducted by performing a Kaplan-Meier estimate, testing for several selected parameters. Cox proportional hazard regression was performed to test the effect of other independent variables on survival times $(1,6,12,24$ months) of different groups of patients, controlling for confounders and interaction terms.

\section{Results}

The baseline clinical and laboratory characteristics of the 195 cirrhotic patients included in the study are summarized in Table 1: 127 patients (65.1\%) were male; the median age was 66 years. The most common cause of cirrhosis was alcohol (36.4\%), followed by viral hepatitis $(28.7 \%)$. One hundred forty-two patients (72.8\%) presented with decompensated cirrhosis. Median MELD, MELD-Na, CP, CP-I, CP-II, ALBI, and PALBI scores were 12 (IQR 9, range 6-30), 15 (IQR 11, range 3-33), 7 (IQR 4, range 5-13), 8 (IQR 5, range 5-17), 7 (IQR 5, range 5-15), -2.68 (IQR 1.23 , range -4.25 to -0.64 ), and -2.55 (IQR 0.91 , range -3.17 to -0.21 ), respectively.

The ROC curves of all 7 scoring systems for 1-, 6-, 12-, and 24-month survival are shown in Fig. 1. All scoring systems were found to have diagnostic accuracy in predicting survival $(\mathrm{P}<0.001)$. ALBI had the optimum balance between sensitivity and specificity (AUC 0.704, 95\%CI 0.630-0.778) compared with
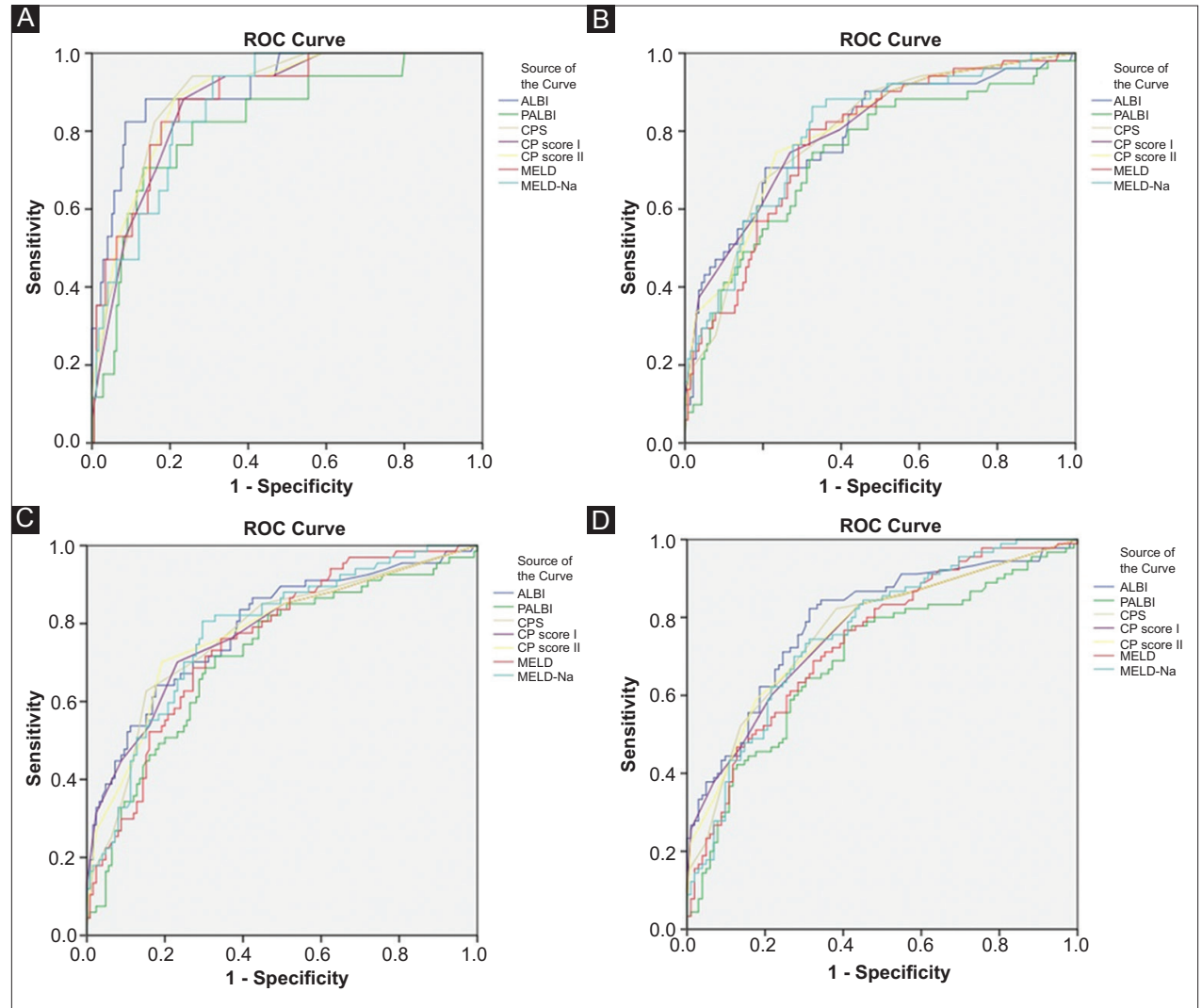

Figure 1 ROC curves CP, modified CP-I, modified CPS-II, MELD, MELD-Na, PALBI and ALBI scores for 1- (A), 6-(B), 12-(C), and 24-(D) months ROC, receiver operating characteristic; CI, confidence interval; CP, Child-Pugh; MELD, model for end-stage liver disease; MELD-Na, model for endstage liver disease with sodium; ALBI, albumin-bilirubin score; PALBI, platelet-albumin-bilirubin score 
the other scores. All scoring systems appeared to have excellent prognostic accuracy (AUC>0.80) for 1-month survival. The ALBI score appeared to have an advantage in predicting 1-, 12-, and 24-month survival (AUC 0.912, 0.781, 0.780, respectively) (Table 2). CP-I and MELD-Na were slightly better in predicting 6-month survival (AUC 0.796 and 0.795, respectively).

When compensated and decompensated cirrhotics were evaluated, none of the scores was satisfactory for survival prognosis; however, the number of compensated cirrhotics at first examination was relatively small and that might have influenced the results. In decompensated cirrhosis the ALBI score was at least similar to, or even better than all the other scores in predicting 1-, 6-, 12- and 24-month survival, as shown in Table 3.

In the univariate analysis, the ALBI, PALBI and MELD$\mathrm{Na}$ scores and age were found to be significantly associated with mortality. Similarly, ALBI and PALBI grade 3 presented at least 14.83 and 2.89 times higher risk, respectively. In the multivariate analysis, the only factors independently associated with death were the ALBI score (hazard ratio [HR] 2.51, 95\% CI 1.69-3.73; $\mathrm{P}<0.001$ ), the MELD-Na score (HR 1.04,
95\% CI 1.00-1.09; $\mathrm{P}=0.045$ ), and age (HR 1.05; 95\%CI 1.03 1.07; $\mathrm{P}<0.001$ ) (Table 4).

In decompensated cirrhotic patients, the multivariate analysis showed that the ALBI score (HR 3.03, 95\%CI 1.924.78; $\mathrm{P}<0.001$ ), and age (HR 1.05, 95\%CI 1.03-1.07; $\mathrm{P}<0.001$ ) were independently associated with death (Table 5).

During the follow-up period 124 patients died. All deaths were liver related. The Kaplan-Meier survival curve indicated a worse prognosis for patients with decompensated cirrhosis $(\mathrm{P}=0.03)$ and for patients with cirrhosis due to alcohol abuse or viral hepatitis $(\mathrm{P}=0.04)$ (Fig. 2).

\section{Discussion}

This study suggests that the ALBI score has a prognostic accuracy similar to that of other established scores for mortality prediction in cirrhosis. These data, if validated in larger studies, may be of significant clinical importance in view of the simplicity of this score.

Table 3 Comparisons of the areas under the ROC curve of CP, CP-I, CP-II, MELD, MELD-Na, ALBI, and PALBI scores for 1-, 6-, 12-, and 24-month survival based on the compensation status

\begin{tabular}{|c|c|c|c|c|c|c|c|}
\hline \multirow{2}{*}{$\begin{array}{l}\text { Survival } \\
\text { (months) }\end{array}$} & \multirow[t]{2}{*}{ Score } & \multicolumn{2}{|c|}{ Area under ROC curve } & \multicolumn{2}{|c|}{$95 \% \mathrm{CI}$} & \multicolumn{2}{|c|}{ P-value } \\
\hline & & Compensated & Decompensated & Compensated & Decompensated & Compensated & Decompensated \\
\hline \multirow[t]{8}{*}{1} & CP score & - & 0.846 & - & $0.76-0.93$ & - & $<0.001$ \\
\hline & CP-I & - & 0.827 & - & $0.73-0.92$ & - & $<0.001$ \\
\hline & CP-II & - & 0.851 & - & $0.76-0.94$ & - & $<0.001$ \\
\hline & MELD & - & 0.32 & - & $0.73-0.93$ & - & $<0.001$ \\
\hline & MELD-Na & - & 0.26 & - & $0.73-0.91$ & - & $<0.001$ \\
\hline & score & - & 0.879 & & $0.78-0.97$ & - & $<0.001$ \\
\hline & ALBI & - & 0.780 & & $0.66-0.89$ & - & $<0.001$ \\
\hline & PALBI & & & & & & \\
\hline \multirow[t]{8}{*}{6} & CP score & 0.536 & 0.755 & $0.23-0.83$ & $0.67-0.83$ & 0.81 & $<0.001$ \\
\hline & CP-I & 0.495 & 0.784 & $0.21-0.77$ & $0.70-0.86$ & 0.97 & $<0.001$ \\
\hline & CP-II & 0.495 & 0.780 & $0.21-0.77$ & $0.70-0.85$ & 0.97 & $<0.001$ \\
\hline & MELD & 0.566 & 0.730 & $0.24-0.89$ & $0.64-0.81$ & 0.66 & $<0.001$ \\
\hline & MELD-Na & 0.561 & 0.770 & $0.28-0.83$ & $0.69-0.84$ & 0.68 & $<0.001$ \\
\hline & score & 0.393 & 0.769 & $0.06-0.71$ & $0.68-0.85$ & 0.48 & $<0.001$ \\
\hline & ALBI & 0.449 & 0.712 & $0.04-0.85$ & $0.62-0.80$ & 0.73 & $<0.001$ \\
\hline & PALBI & & & & & & \\
\hline \multirow[t]{7}{*}{12} & CP score & 0.469 & 0.764 & $0.26-0.67$ & $0.68-0.84$ & 0.78 & $<0.001$ \\
\hline & CP-I & 0.431 & 0.792 & $0.23-0.62$ & $0.71-0.86$ & 0.53 & $<0.001$ \\
\hline & CP-II & 0.431 & 0.791 & $0.23-0.62$ & $0.71-0.86$ & 0.53 & $<0.001$ \\
\hline & MELD & 0.683 & 0.716 & $0.48-0.88$ & $0.63-0.80$ & 0.10 & $<0.001$ \\
\hline & MELD-Na & 0.617 & 0.772 & $0.44-0.79$ & $0.69-0.84$ & 0.29 & $<0.001$ \\
\hline & ALBI & 0.517 & 0.782 & $0.27-0.75$ & $0.70-0.85$ & 0.88 & $<0.001$ \\
\hline & PALBI & 0.494 & 0.702 & $0.22-0.76$ & $0.61-0.78$ & 0.96 & $<0.001$ \\
\hline \multirow[t]{7}{*}{24} & CP score & 0.441 & 0.749 & $0.26-0.61$ & $0.66-0.83$ & 0.53 & $<0.001$ \\
\hline & CP-I & 0.458 & 0.760 & $0.27-0.63$ & $0.68-0.83$ & 0.66 & $<0.001$ \\
\hline & CP-II & 0.455 & 0.768 & $0.27-0.63$ & $0.69-0.84$ & 0.64 & $<0.001$ \\
\hline & MELD & 0.636 & 0.698 & $0.45-0.81$ & $0.61-0.78$ & 0.15 & $<0.001$ \\
\hline & MELD-Na & 0.608 & 0.735 & $0.45-0.76$ & $0.65-0.81$ & 0.26 & $<0.001$ \\
\hline & ALBI & 0.477 & 0.786 & $0.27-0.67$ & $0.71-0.86$ & 0.80 & $<0.001$ \\
\hline & PALBI & 0.374 & 0.697 & $0.16-0.58$ & $0.61-0.78$ & 0.18 & $<0.001$ \\
\hline
\end{tabular}

ROC, receiver operating characteristic; CI, confidence interval; CP, Child-Pugh; MELD, model for end-stage liver disease; MELD-Na, model for end-stage liver disease with sodium; ALBI, albumin-bilirubin score; PALBI, platelet-albumin-bilirubin score 
Table 4 Crude and adjusted HR for death and 95\%CI for ALBI, PALBI and MELD-Na scores

\begin{tabular}{|c|c|c|c|c|c|c|}
\hline \multirow[t]{2}{*}{ Covariates } & \multicolumn{3}{|c|}{ Unadjusted } & \multicolumn{3}{|c|}{ Adjusted $^{\mathrm{a}}$} \\
\hline & HR $(95 \% \mathrm{CI})$ & c-statistics HR & P-value & HR $(95 \% \mathrm{CI})$ & c-statistics HR & P-value \\
\hline ALBI & $\begin{array}{c}2.78 \\
(2.11-3.67)\end{array}$ & 0.704 & $<0.001$ & $\begin{array}{c}2.51 \\
(1.69-3.73)\end{array}$ & 0.704 & $<0.001$ \\
\hline PALBI & $\begin{array}{c}2.16 \\
(1.62-2.89)\end{array}$ & 0.695 & $<0.001$ & - & - & - \\
\hline MELD-Na & $\begin{array}{c}1.11 \\
(1.07-1.14)\end{array}$ & 0.683 & $<0.001$ & $\begin{array}{c}1.04 \\
(1.00-1.09)\end{array}$ & 0.683 & 0.045 \\
\hline Age & $\begin{array}{c}1.03 \\
(1.01-1.05)\end{array}$ & 0.641 & $<0.001$ & $\begin{array}{c}1.05 \\
(1.03-1.07)\end{array}$ & 0.641 & $<0.001$ \\
\hline \multicolumn{7}{|l|}{ ALBI grade } \\
\hline 1 & 1 & - & - & - & - & - \\
\hline 2 & $\begin{array}{c}2.42 \\
(1.65-3.53)\end{array}$ & 0.701 & $<0.001$ & - & - & - \\
\hline 3 & $\begin{array}{c}14.83 \\
(7.67-28.78)\end{array}$ & 0.942 & $<0.001$ & - & - & - \\
\hline \multicolumn{7}{|c|}{ PALBI grade } \\
\hline 1 & 1 & - & - & - & - & - \\
\hline 2 & $\begin{array}{c}1.40 \\
(0.86-2.26)\end{array}$ & 0.686 & 0.16 & - & - & - \\
\hline 3 & $\begin{array}{c}2.89 \\
(1.93-4.32)\end{array}$ & 0.811 & $<0.001$ & - & - & - \\
\hline
\end{tabular}

${ }^{\mathrm{a} M u l t i v a r i a t e}$ model adjusted for sex

$H R$, hazard ratio; CI, confidence interval; MELD, model for end-stage liver disease; $M E L D-N a$, model for end-stage liver disease with sodium; ALBI, albumin-bilirubin score; PALBI, platelet-albumin-bilirubin score

Table 5 Crude and adjusted HR for death and 95\%CI for ALBI score in decompensated cirrhotic patients

\begin{tabular}{|c|c|c|c|c|c|c|}
\hline \multirow[t]{2}{*}{ Covariates } & \multicolumn{3}{|c|}{ Unadjusted } & \multicolumn{3}{|c|}{ Adjusted $^{\mathrm{a}}$} \\
\hline & HR $(95 \% \mathrm{CI})$ & c-statistics HR & P-value & HR $(95 \% \mathrm{CI})$ & c-statistics HR & P-value \\
\hline ALBI & $\begin{array}{c}3.33 \\
(2.32-4.77)\end{array}$ & 0.704 & $<0.001$ & $\begin{array}{c}3.03 \\
(1.92-4.78)\end{array}$ & 0.704 & $<0.001$ \\
\hline Age & $\begin{array}{c}1.04 \\
(1.02-1.06)\end{array}$ & 0.641 & $<0.001$ & $\begin{array}{c}1.05 \\
(1.03-1.07)\end{array}$ & 0.641 & $<0.001$ \\
\hline
\end{tabular}

${ }^{\mathrm{a} M u l t i v a r i a t e}$ model adjusted to sex

ALBI, albumin-bilirubin score; MELD-Na, model of end-stage liver disease with sodium score
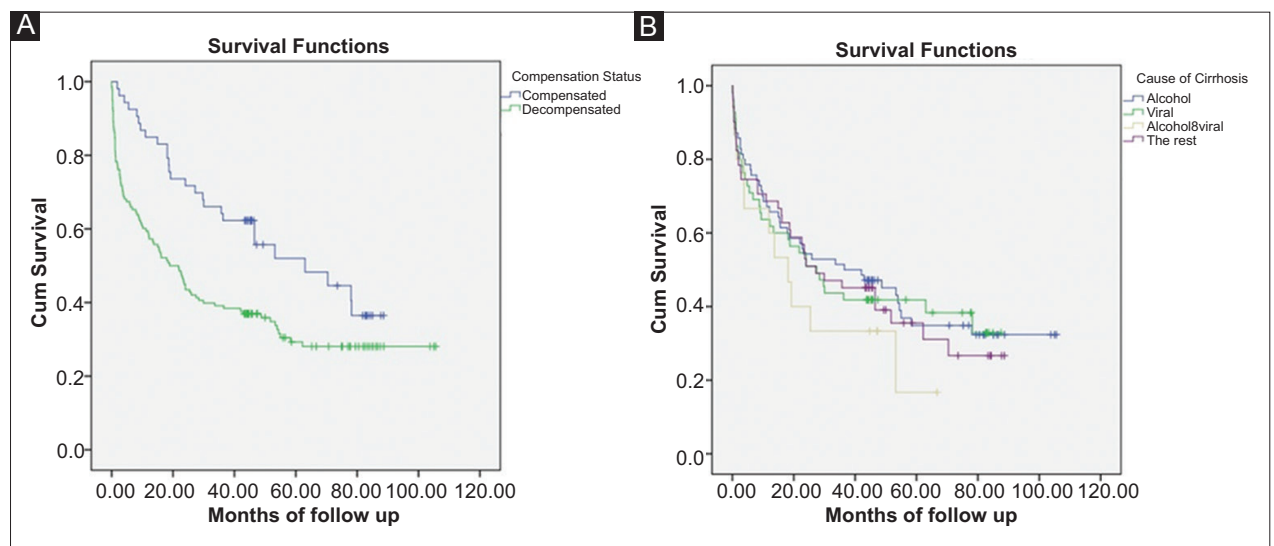

Figure 2 Kaplan-Meier survival curve for patients according to their compensation status (A) and the etiology of cirrhosis (B) 
The CP score has been proposed as a reliable prognostic score in cirrhosis in many systematic reviews [19-21], but its subjective components (ascites and encephalopathy) still remain a matter of doubt. A systematic review of prognostic indicators in cirrhosis in 118 studies showed that serum albumin and bilirubin were the 2 most significant prognostic variables for survival [21].

A study by Chen et al [14] assessed the accuracy of ALBI in predicting 1-, 2-, and 3- year mortality in patients with hepatitis B-related cirrhosis, and ROC curves showed that the ALBI score (AUC 0.787, 0.830, and 0.833) was superior to the $\operatorname{MELD}(0.693, \mathrm{P}=0.003 ; 0.717, \mathrm{P}<0.001 ; 0.744, \mathrm{P}<0.001)$ and CP $(0.641, \mathrm{P}<0.001 ; 0.649, \mathrm{P}<0.001 ; 0.657, \mathrm{P}<0.001)$ scores. In our study, in agreement with Chen et al [14], ROC analysis showed that the ALBI score was better at predicting mortality compared to MELD, MELD-Na, CP, CP-I and CP-II scores. Moreover, in the aforementioned study [14] the multivariate analysis found ALBI score and age to be independent factors associated with mortality, in accordance with our study, while in another study that included only patients with primary biliary cholangitis [12] the multivariate analysis also indicated that the ALBI score was the only independent prognostic factor, with an adjusted HR for developing a hepatic event of $27.8(\mathrm{P}<0.001)$. These findings agree with our study, where ALBI, MELD-Na score, and age were all independent variables, whereas in decompensated cirrhosis only the ALBI score was an independent prognostic factor. A study by Zou et al [13] evaluated the in-hospital mortality in relation to ALBI, CP, and MELD scores in 631 cirrhotic patients and found that the ALBI score had the best AUC (0.808, 0.785, 0.787, respectively). These studies suggest that ALBI is a good indicator of shortterm prognosis, in agreement with our study, where 1-month survival was best assessed by ALBI (AUC 0.912).

Another study evaluated CP score, MELD, and ALBI in predicting 3-month mortality in patients with acute-on-chronic liver failure and reported that both ALBI and MELD scores were independent predictors $(\mathrm{P}<0.001)$; however, the ROC curves indicated that the MELD score was better than the ALBI score (AUC 0.837 and 0.784, respectively) [15]. Furthermore, a study from Taiwan in 242 patients with both compensated and decompensated cirrhosis concluded that both ALBI and MELD scores significantly predicted 3-month and 6-month mortality (AUC 0.773, 0.691 vs. 0.813, 0.740, respectively) [22].

Moreover, a recent, prospective study validated the ALBI score as a measure of liver dysfunction in patients with compensated cirrhosis and proposed a new score, ALBIFIB4, to stratify these patients for the risk of future liver decompensation [23]. In addition, the ALBI score has been found to significantly predict portal hypertension (both clinically significant and severe) [22].

A study by Oikonomou et al [17] assessed the grades of both ALBI and PALBI scores in patients with decompensated cirrhosis and found that both ALBI and PALBI grade 3 patients had at least double the risk of death or transplantation. Our results are in accordance with the aforementioned study and outline the importance of ALBI grade 3, as it showed a 14 times higher risk of death compared with a 2.42 times higher risk for ALBI grade 2 .

\section{Summary Box}

\section{What is already known:}

- The existence of reliable prognostic indices is of paramount importance in the management of cirrhosis

- The albumin-bilirubin (ALBI) score is a recently reported very simple score, initially used in hepatocellular carcinoma, which has not been thoroughly investigated in cirrhosis

\section{What the new findings are:}

- The ALBI score is an overall accurate and reliable score in decompensated cirrhosis, irrespective of etiology, equal to or better than the other predictive scores in predicting mortality

- Given its simplicity, the ALBI score can confidently replace the other more complex scores in addition to hepatocellular carcinoma prognosis

Our study has the limitation that it was conducted in a single tertiary institute with a relative small number of cases. Therefore, a larger cohort of well stratified cirrhotics of different etiologies should be studied in order to confirm our findings. Moreover, there was an imbalance in the compensation status of our patients, as more than $70 \%$ already had decompensated cirrhosis when they were enrolled in our study.

Nonetheless, our study has demonstrated that ALBI is an overall accurate and reliable score in decompensated cirrhosis, irrespective of etiology, equal to or better than the other predictive scores in predicting mortality. Therefore, in view of its simplicity, it can confidently replace the other more complex scores.

\section{References}

1. Child CG, Turcotte JG. Surgery and portal hypertension. Major Probl Clin Surg 1964;1:1-85.

2. Pugh RN, Murray-Lyon IM, Dawson JL, Pietroni MC, Williams R. Transection of the oesophagus for bleeding oesophageal varices. $\mathrm{Br}$ J Surg 1973;60:646-649.

3. Child CG, Turcotte JG. Surgery and portal hypertension. In: Child CG (editor): The liver and portal hypertension. Saunders: Philadelphia; 1964, pp. 50-64.

4. Angermayr B, Koenig F, Cejna M, et al. Creatinine-modified Child-Pugh score (CPSC) compared with MELD-score to predict survival in patients undergoing TIPS. Hepatology 2002;36:378A.

5. Malinchoc M, Kamath PS, Gordon FD, Peine CJ, Rank J, ter Borg PC. A model to predict poor survival in patients undergoing transjugular intrahepatic portosystemic shunts. Hepatology 2000;31:864-871.

6. Wiesner R, Edwards E, Freeman R, et al; United Network for 
Organ Sharing Liver Disease Severity Score Committee. Model for end-stage liver disease (MELD) and allocation of donor livers. Gastroenterology 2003;124:91-96.

7. Londoño MC, Cárdenas A, Guevara $\mathrm{M}$, et al. MELD score and serum sodium in the prediction of survival of patients with cirrhosis awaiting liver transplantation. Gut 2007;56:1283-1290.

8. Biggins SW, Rodriguez HJ, Bacchetti P, Bass NM, Roberts JP, Terrault NA. Serum sodium predicts mortality in patients listed for liver transplantation. Hepatology 2005;41:32-39.

9. Biggins SW, Kim WR, Terrault NA, et al. Evidence-based incorporation of serum sodium concentration into MELD. Gastroenterology 2006;130:1652-1660.

10. Toyoda H, Lai PB, O’Beirne J, et al. Long-term impact of liver function on curative therapy for hepatocellular carcinoma: application of the ALBI grade. Br J Cancer 2016;114:744-750.

11. Johnson PJ, Berhane S, Kagebayashi C, et al. Assessment of liver function in patients with hepatocellular carcinoma: a new evidencebased approach-the ALBI grade. J Clin Oncol 2015;33:550-558.

12. Chan AW, Chan RC, Wong GL, et al. New simple prognostic score for primary biliary cirrhosis: Albumin-bilirubin score. J Gastroenterol Hepatol 2015;30:1391-1396.

13. Zou D, Qi X, Zhu C, et al. Albumin-bilirubin score for predicting the in-hospital mortality of acute upper gastrointestinal bleeding in liver cirrhosis: A retrospective study. Turk J Gastroenterol 2016;27:180-186.

14. Chen RC, Cai YJ, Wu JM, et al. Usefulness of albumin-bilirubin grade for evaluation of long-term prognosis for hepatitis B-related cirrhosis. J Viral Hepat 2017;24:238-245.

15. Chen B, Lin S. Albumin-bilirubin (ALBI) score at admission predicts possible outcomes in patients with acute-on-chronic liver failure. Medicine (Baltimore) 2017;96:e7142.

16. Kamath PS, Wiesner RH, Malinchoc M, et al. A model to predict survival in patients with end-stage liver disease. Hepatology 2001;33:464-470.

17. Oikonomou T, Goulis L, Doumtsis P, Tzoumari T, Akriviadis E, Cholongitas E. ALBI and PALBI Grades Are Associated with the Outcome of Patients with Stable Decompensated Cirrhosis. Ann Hepatol 2019;18:126-136.

18. Chedid MF, Picon RV, Chedid AD. ALBI and PALBI: Novel Scores for Outcome Prediction of Cirrhotic Outpatients Awaiting Liver Transplantation. Ann Hepatol 2018;17:906-907.

19. Cholongitas E, Papatheodoridis GV, Vangeli M, Terreni N, Patch D, Burroughs AK. Systematic review: The model for end-stage liver disease-should it replace Child-Pugh's classification for assessing prognosis in cirrhosis? Aliment Pharmacol Ther 2005;22:1079-1089.

20. Cholongitas E, Marelli L, Shusang V, et al. A systematic review of the performance of the model for end-stage liver disease (MELD) in the setting of liver transplantation. Liver Transpl 2006;12:1049-1061.

21. D’Amico G, Garcia-Tsao G, Pagliaro L. Natural history and prognostic indicators of survival in cirrhosis: a systematic review of 118 studies. J Hepatol 2006;44:217-231.

22. Hsieh YC, Lee KC, Wang YW, et al. Correlation and prognostic accuracy between noninvasive liver fibrosismarkers and portal pressure in cirrhosis: Role of ALBI score. PLoS One 2018;13:e0208903.

23. Guha IN, Harris R, Berhane S, et al. Validation of a model for identification of patients with compensated cirrhosis at high risk of decompensation. Clin Gastroenterol Hepatol 2019 [Epub ahead of print]. doi: 10.1016/j.cgh.2019.01.042 